yields a neutral test, the boiling has to be prolonged in every analysis-for what purpose? Merely to save the trouble of weighing a quantity of carbonate into a measured bulk of water. Here the trouble certainly surpasses the saving at the production of the test solution.

The nature of the analyses for uric acid also helps to strengthen my resolution here publicly to assure Dr.Jones that such analyses are of no use to pathology, to patients, or to readers. If such are to be the fruits which medicine is to ripen from the flowers of chemistry (and I believe both Liebig's and Davy's methods to be flowers of that science, the one by its profound shrewdness, the other by its great simplicity), we had much rather beg chemists not to trouble themselves in our behalf. We have long been sighing for a method to analyse urea and determine its quantity. Scarcely are we presented with a good one, when up start a series of incompetent critics and dissatisfied reformers, the first to find a thousand fallacies where nothing is fallacious but themselves, the others to deform by their intended reformation. I have heard Liebig's method declared to be fallacious by men who had never as much as read it, and who were not only utterly incompetent to try it, but also so destitute of the elements of chemistry as confidently to pronounce nitrate of baryta the best means for absorbing carbonic acid. To be sure, the best of us are liable to error, but always open to correction; it is, however, difficult to cure errors in principle. Our present morals in medical chemistry are rather lax as regards quantities. My intention in this article has been to contribute towards making them a little more stringent.

\section{CASE OF RHEUMATIC PARALYSIS.}

By Benjamin Dulley, Esq., F.R.C.S., Wellingborough.

$$
\text { [Read before the South Midland Branch, May 21, 1857.] }
$$

THE patient was a man, E. S., aged 32 , married, living at Wellingborough, in a somewhat damp house, but in a very healthy situation. He was by trade a shoemaker, and up to January 1856, had always, with slight intermissions, enjoyed good health. On the 16th of that month, he was attacked with severe pain in the bowels, which were tender and con stipated: but he was soon relieved by purgatives, combined with calomel and opium.

On February 3rd, he began to complain of acute pain in the left shoulder and elbow-joints, accompanied by heat, thirst, and restlessness. On the second day, the pain extended to the left arm, then to both hands and wrist-joints, which were slightly swollen. In about a week or ten days the severity of the symptoms had in some degree ceased, but then the pain was felt extending down the back to the hips, knees, and ankles. Again, the febrile symptoms were renewed; the tongue became furred, the pulse quick, the urine scanty, and with bright red deposit; the breathing rapid and jerking; and he had occasional delirium. This state continued for six weeks. The treatment consisted of antimony, opium, and small doses of calomel; afterwards, when the lower limbs became affected, colchicum with bicarbonate of potash was added to the other remedies with marked benefit. The febrile symptoms gra dually subsided, but unfortunately left the patient weak and incapable of the slightest movement. Paralysis seemed complete in all the voluntary muscles; but the patient retained his power over the rectum and bladder. Week after week now passed with no amendment; blisters and other irritants were applied to the spine; the patient took quinine, ammonia, iron, iodide of potassium, and zinc, in succession, and had the continued daily use of electro-galvanism up to June 21st. At this time, he had only so far recovered as to be able to move his neck freely, the right arm stiffly, and in some degree the fingers of the right hand. At this date, he was carefully conveyed to the Northampton Infirmary, and there most assiduously attended by Dr. Webster (who took a great interest in the case) for four months, but returned with his paralytic symptoms unalleviated.

At the present time (May 21st, 1857), more than fifteen months from the commencement of the attack, his health is good; he eats and drinks well; indeed, he gets fat and looks cheerful; he can move his head in all directions; he has the power when in bed of rolling from the left side on to his tack, which is a great relief; he can move the right arm, but the hand is drawn back and incapable of much flexion; with difficulty he can grasp a fork, but is unable to feed himself. The left arm lies by his side useless; nor can the hand be flexed or brought up to the head. The right leg he can move freely when lying on the left side, but cannot draw it upwards. The left leg is useless. There is no swelling in any of the joints. $\mathrm{He}$ has no difficulty in passing either urine or fæces: the former is of healthy character and sufficient quantity. The bowels are generally moved once a day.

He has never had any loss of feeling; and has rather an exquisite sensitiveness to the least touch throughout the whole body.

ReMarks. There is no novelty in rheumatic paralysis; and the present case was introduced to the attention of the meeting for the purpose of inviting discussion as to the nature and causes of paralysis in rheumatic fever. It was also desired to educe the practical experience of those members who had met with cases similar to that of my unfortunate patient; and to ascertain if any plan of treatment had been devised for alleviating so great a calamity. It was suggested that a general long continued use of the iodide of potassium was an important and frequently a successful remedy; as was also the frequent application of blisters to the spine, combined with attention to the state of the health generally. These remedies, as well as others mentioned above, had been fairly tried, and, I am sorry to say, had utterly failed.

\section{EFFECTS OF FULL DOSES OF OPIUM.}

By Frederick Pritchard, Esq., Stratford-upon-Avon.

CASE I. About ten years since, my father and my assistant requested me to visit with them a person of the name of $\mathbf{J}$. C., aged 33 , of Shottery, in this neighbourhood, who had been suffering from intussusceptio for nine days. The symptoms were most violent, and accompanied with hiccup, stercoraceous vomiting, subsultus, and every sign of approaching dissolution. The usual remedies had been resorted to without any beneficial effect. I prescribed four grains of solid opium to be administered in the form of a pill. In twelve hours the bowels acted, and the patient speedily recovered, and has continued well from that period.

CASE II. Five years afterwards, my assistant requested me to visit another case with him. The patient, L. R., aged 50, residing at Clifford Chambers, as in Case I, had very nearly the same symptoms, and had been ill for several days before she applied. The same quantity of opium was administered, and on the following day she was relieved, and very soon recovered.

CASE III. Six years since, I was called to a lady, aged 63 , with strangulated umbilical hernia, an irreducible one of long standing. I employed the ordinary taxis, and also resorted to the usual means for the purpose of relieving the strangulation, but without effect: vomiting of stercoraceous matter supervened, and the patient appeared to be sinking rapidly. A hospital surgeon was called in consultation; and it was agreed that, in consequence of the severity of the symptoms, as well as the exhausted condition of the patient, an operation was out of the question. The same quantity of opium I shortly afterwards administered, and with the same happy effect, for the bowels acted in the course of ten hours, and she rapidly recovered.

I have given a very brief but truthful sketch of these casescoses in which I hove felt much interested; and $\mathrm{my}$ opinion is, that in each the large dose of opium, by acting as a direct sedative, so unstrung (if I may be allowed the term) the bowel, as at once to relieve the intestinal obstruction.

The following case having occurred at the time I was thinking of sending the preceding ones for insertion, I waited to see the result of it; and as so much has been said and published lately respecting idiopathic tetanus, any contribution, I imagine, will be read with interest.

CaSE IV. On August 22nd, at 8 o'clock A.M., my assistant requested me to visit a person of the name of Richard Brown, aged 27 , who he stated was suffering from tetanus, but he could not ascribe the affection to any particular cause. $\mathrm{He}$ informed me that when he first saw the patient, he found him in a state of great exhaustion; the pulse was small and fluttering; the extremities cold; and with these symptoms were tetanic convulsions. He prescribed ammonia, opium, and chloric æther, in full doses, and gave the patient a pint of brandy in the course of two hours.

When I visited him, reaction to a certain extent had come on; his pulse was firm, and the state of the skin improved, but the tetanic convulsions had very much increased in severity. 